Real Analysis Exchange

Vol. 29(2), 2003/2004, pp. 921-930

M. Balcerzak, Institute of Mathematics, Technical University of Łódź, ul. Wólczańska 215, 93-005 Łódź, Poland, and Faculty of Mathematics, Łódź University, Banacha 22, 90-238 Łódź, Poland. email: mbalce@p.lodz.pl

A. Kucia, Department of Mathematics, Silesian University, 40-007 Katowice, Poland. email: kucia@ux2.math.us.edu.pl

A. Nowak, Department of Mathematics, Silesian University, 40-007

Katowice, Poland. email: anowak@ux2.math.us.edu.pl

\title{
REGULAR DEPENDENCE OF TOTAL VARIATION ON PARAMETERS
}

\begin{abstract}
Let $X$ be an interval, $Y$ a metric space, $T$ a set of parameters, and $f: T \times X \rightarrow Y$ a function. For given $t \in T$ denote by $v(t)$ the total variation of $f(t, \cdot)$ on $X$. We look for sufficient conditions for regular (measurable, continuous, etc.) dependence of $v$ on $t$.
\end{abstract}

\section{Preliminaries}

Throughout this paper $X$ is an interval (open, closed, half-closed, bounded or not) on the real line, and $(Y, d)$ is a metric space. Given a mapping $g: X \rightarrow Y$ we define the total variation of $g$ on $X$ as

$$
V(g, X)=\sup _{\Pi} \sum_{i=1}^{n} d\left(g\left(x_{i}\right), g\left(x_{i-1}\right)\right),
$$

where the supremum is taken over all partitions $\Pi=\left\{x_{0}, x_{1}, \ldots, x_{n}\right\}$ of $X$ (i.e., $n \in \mathbb{N}, x_{0}<x_{1}<\cdots<x_{n}$ and $\left.x_{i} \in X, i=0,1, \ldots, n\right)$. We say that $g$ is of bounded variation if $V(g, X)<\infty$.

Let $T$ be a nonempty set of parameters and $f: T \times X \rightarrow Y$ a mapping. Let $v: T \rightarrow[0, \infty]$ be given by $v(t)=V(f(t, \cdot), X)$, i.e., $v(t)$ is the total variation of $f(t, \cdot)$ on $X$. We shall look for sufficient conditions for regular (measurable, continuous, etc.) dependence of $v$ on $t$. This problem appeared in the

Key Words: function of bounded variation, measurability, continuity.

Mathematical Reviews subject classification: Primary 26A45; Secondary 26B99.

Received by the editors July 29, 2003

Communicated by: B. S. Thomson 
study of regular selectors for multifunctions of bounded variation depending on parameters $([2])$.

Let $Z$ be a topological space. We say that $Z$ is Polish if it is separable and can be metrizable by a complete metric. By $\mathcal{B}(Z)$ we denote the Borel $\sigma$-field on $Z$. Suppose $T$ is endowed with a $\sigma$-field $\mathcal{T}$. Then $\mathcal{T} \otimes \mathcal{B}(Z)$ denotes the product $\sigma$-field on $T \times Z$.

We shall need some further terminology. Denote by $\mathcal{N}$ and $\mathcal{N}^{*}$, respectively, the sets of infinite and finite sequences of positive integers. Let $\mathcal{F}$ be a family of sets. We say that $A$ is obtained from $\mathcal{F}$ by the Suslin operation if

$$
A=\bigcup_{\sigma \in \mathcal{N}} \bigcap_{n=1}^{\infty} F\left(\sigma_{1}, \ldots, \sigma_{n}\right)
$$

for some function $F: \mathcal{N}^{*} \rightarrow \mathcal{F}$. Denote by $S(\mathcal{F})$ the family of all sets obtained from $\mathcal{F}$ by the Suslin operation. The family $\mathcal{F}$ is closed with respect to the Suslin operation if $S(\mathcal{F})=\mathcal{F}($ cf. [4],[6])

Recall that a $\sigma$-field $\mathcal{T}$ is closed with respect to the Suslin operation provided one of the following conditions is satisfied: (i) $\mathcal{T}$ is complete with respect to a $\sigma$-finite measure; (ii) $T$ is a topological space and $\mathcal{T}$ is the Baire $\sigma$-field, i.e., $\mathcal{T}$ is the family of all subsets of $T$ having the Baire property; (iii) $T$ is a locally compact space and $\mathcal{T}$ is the family of all subsets of $T$ measurable with respect to a Radon measure (see e.g. [4],[6]).

Let $A$ be a subset of $T \times Z$. Given $t \in T$, we denote by $A_{t}$ the $t$-section of $A$; i.e., $A_{t}=\{z:(t, z) \in A\}$. By $\operatorname{Proj}_{T} A$ we mean the projection of $A$ on $T$; i.e., $\operatorname{Proj}_{T} A=\{t \in T:(t, z) \in A$ for some $z \in Z\}$.

Let $\mathcal{T}$ be a $\sigma$-field on $T$ and $A \in \mathcal{T} \otimes \mathcal{B}(Z)$. In general, $\operatorname{Proj}_{T} A$ does not belong to $\mathcal{T}$. We shall use the following well known results:

Projection Theorem. (see e.g. [4, Theorem 1.3]) If $(T, \mathcal{T})$ is a measurable space, $Z$ is a Polish space and $A \in \mathcal{T} \otimes \mathcal{B}(Z)$, then $\operatorname{Proj}_{T} A \in S(\mathcal{T})$.

Arsenin-Kunugui-Novikov Theorem. (e.g. [6, Theorem 18.18]) Suppose $T$ and $Z$ are Polish spaces, and $A \in \mathcal{B}(T \times Z)$ has $\sigma$-compact t-sections. Then $\operatorname{Proj}_{T} A \in \mathcal{B}(T)$.

\section{Measurability}

Throughout this section $(T, \mathcal{T})$ is a measurable space, $(Y, d)$ is a separable metric space, $f: T \times X \rightarrow Y$ is a function, and $v$ is the total variation of $f(t, \cdot)$ on $X$. We are interested in the measurability properties of the function $v$. Let us start with some examples, where $T=X=Y=[0,1]$. 
Example 2.1. Lebesgue measurable function $f:[0,1]^{2} \rightarrow[0,1]$ such that $v$ is not Lebesgue measurable.

Let $A$ be a nonmeasurable subset of $(0,1), B=\{(t, x): t=x \in A\}$, and $f=\chi_{B}$, the characteristic function of $B$. Clearly, $f$ is Lebesgue measurable, and $v(t)=2$ for $t \in A$ and $v(t)=0$ for $t \notin A$.

Example 2.2. Function $f$ measurable with respect to the Baire $\sigma$-field on $[0,1]^{2}$ such that $v$ is not Baire measurable.

This is an obvious modification of the previous example with $A \subset(0,1)$ without the Baire property.

Example 2.3. Borel measurable function $f:[0,1]^{2} \rightarrow[0,1]$ such that $v$ is not Borel measurable.

Let $P$ be the set of all irrationals from $[0,1]$, and $A \subset[0,1]$ an analytic non-Borel set. There is a continuous and onto function $h: P \rightarrow A$. Let $D=\{(h(x), x): x \in P\}$; i.e., $D$ is the graph of $h$. Since $h$ is continuous, $D$ is a closed subset of $[0,1] \times P$ and, consequently, a Borel subset of $[0,1]^{2}$. Let $f=\chi_{D}$. Then $v(t) \geq 2$ for $t \in A$ and $v(t)=0$ for $t \notin A$. Hence, $v$ is not Borel measurable.

Since $\mathcal{B}\left([0,1]^{2}\right)=\mathcal{B}([0,1]) \otimes \mathcal{B}([0,1])$, the last example shows that if $f$ is $\mathcal{T} \otimes \mathcal{B}(X)$-measurable, then $v$ need not be $\mathcal{T}$-measurable.

We start with a theorem which gives sufficient conditions for the measurability of the total variation for an arbitrary measurable space $(T, \mathcal{T})$.

Theorem 2.1. Suppose there exists a countable dense subset $E$ of $X$ such that the following conditions are satisfied:

(i) For each $(t, x) \in T \times X$ the value $f(t, x)$ is the limit of $\left(f\left(t, e_{k}\right)\right)$ for some sequence $\left(e_{k}\right)$ of points of $E$ convergent to $x$.

(ii) For each $e \in E, f(\cdot, e)$ is measurable.

Then $v$ is measurable.

Proof. Fix $t \in T$. For each partition $\Pi=\left\{x_{0}, \ldots, x_{n}\right\}$ of $X$ and each $\varepsilon>0$ there exists a partition $\Pi^{\prime}=\left\{x_{0}^{\prime}, \ldots, x_{n}^{\prime}\right\}$ consisting of points of $E$, such that

$$
\sum_{i=1}^{n} d\left(f\left(t, x_{i}\right), f\left(t, x_{i-1}\right)\right) \leq \varepsilon+\sum_{i=1}^{n} d\left(f\left(t, x_{i}^{\prime}\right), f\left(t, x_{i-1}^{\prime}\right)\right) .
$$

Hence,

$$
v(t)=\sup _{\Pi^{\prime}} \sum_{i=1}^{n} d\left(f\left(t, x_{i}^{\prime}\right), f\left(t, x_{i-1}^{\prime}\right)\right), t \in T,
$$


where supremum is taken over all partitions of $X$ consisting of elements of $E$. Under our assumptions, for each such a partition $\Pi^{\prime}$ the function

$$
t \mapsto \sum_{i=1}^{n} d\left(f\left(t, x_{i}^{\prime}\right), f\left(t, x_{i-1}^{\prime}\right)\right), t \in T
$$

is measurable. Thus $v$ is measurable, as the pointwise supremum of a countable family of measurable functions.

Remark 2.1. We list some cases, when the condition (i) of the last theorem is satisfied for each dense subset $E \subset X$ :

1. $f$ is continuous in $x$.

2. $f$ is one-sided continuous in $x$ (possibly, left-continuous at some points and right-continuous at others).

3. $f$ is quasi-continuous in $x$ (see e.g. [9]).

We shall use the following technical lemmas.

Lemma 2.1. For any $r \geq 0$ we have $\{t \in T: v(t)>r\}=\bigcup\left\{\operatorname{Proj}_{T} A_{n}: n \in\right.$ $\mathbb{N}\}$, where

$$
\begin{aligned}
A_{n}=\left\{\left(t, x_{0}, \ldots, x_{n}\right) \in T \times X^{n+1}:\right. & x_{0}<x_{1}<\cdots<x_{n} \\
& \left.\sum_{i=1}^{n} d\left(f\left(t, x_{i}\right), f\left(t, x_{i-1}\right)\right)>r\right\} .
\end{aligned}
$$

Proof. Indeed, $v(t)>r$ iff there is a partition $\left\{x_{0}, x_{1}, \ldots, x_{n}\right\}$ of $X$ such that $\sum_{i=1}^{n} d\left(f\left(t, x_{i}\right), f\left(t, x_{i-1}\right)\right)>r$.

Lemma 2.2. If $f$ is $\mathcal{T} \otimes \mathcal{B}(X)$-measurable, then $A_{n} \in \mathcal{T} \otimes \mathcal{B}\left(X^{n+1}\right)$.

Proof. Let an auxiliary function $g: T \times X^{n+1} \rightarrow \mathbb{R}$ be defined by

$$
g\left(t, x_{0}, \ldots, x_{n}\right)=\sum_{i=1}^{n} d\left(f\left(t, x_{i}\right), f\left(t, x_{i-1}\right)\right) .
$$

Since $Y$ is separable, $g$ is $\mathcal{T} \otimes \mathcal{B}\left(X^{n+1}\right)$-measurable. Now the measurability of $A_{n}$ follows from $A_{n}=T \times U_{n} \cap g^{-1}((r, \infty))$, where $U_{n}=\left\{x \in X^{n+1}: x_{0}<\right.$ $\left.\cdots<x_{n}\right\}$ is open in $X^{n+1}$.

Theorem 2.2. If $f$ is $\mathcal{T} \otimes \mathcal{B}(X)$-measurable then $v$ is $\sigma(S(\mathcal{T}))$-measurable. 
Proof. Fix $r \geq 0$. By Lemma 2.2, $A_{n} \in \mathcal{T} \otimes \mathcal{B}\left(X^{n+1}\right), n \in \mathbb{N}$. Since $X^{n+1}$ is a Polish space, we can use the Projection Theorem. Thus $\operatorname{Proj}_{T} A_{n} \in S(\mathcal{T})$ and, consequently, $v$ is measurable with respect to the $\sigma$-field generated by $S(\mathcal{T})$.

Corollary 2.1. If the $\sigma$-field $\mathcal{T}$ is closed under the Suslin operation and $f$ is $\mathcal{T} \otimes \mathcal{B}(X)$-measurable, then $v$ is $\mathcal{T}$-measurable.

Corollary 2.2. Suppose $T$ is a Lebesgue measurable subset of $\mathbb{R}^{m}$ and $\mathcal{T}=$ $\mathcal{L}_{m}(T)$. If $f$ is $\mathcal{L}_{m}(T) \otimes \mathcal{B}(X)$-measurable, then $v$ is Lebesgue measurable.

Corollary 2.3. If $T$ is a Polish space and $f$ is Borel measurable, then $v$ is measurable with respect to the $\sigma$-field generated by analytic subsets of $T$.

Remind that a function $h: Z \rightarrow Y$, where $Z$ is metrizable, is of the $1 s t$ class of Baire if $h^{-1}(U) \in F_{\sigma}(Z)$ for each open $U \subset Y$. If $h$ is the pointwise limit of a sequence of continuous functions, then it is of the 1st class of Baire. If $Y$ is an interval, then these two conditions are equivalent. The function $h$ is of the 2nd class of Baire if for each open $U \subset Y, h^{-1}(U) \in G_{\delta \sigma}(Z)$.

Theorem 2.3. Suppose $T$ is a Polish space, $f: T \times X \rightarrow Y$ is Borel measurable, and for each $t \in T, f(t, \cdot)$ is of the 1st class of Baire. Then $v$ is Borel measurable.

Proof. Since $Y$ is separable, for fixed $t$ the function

$$
\left(x_{0}, \ldots, x_{n}\right) \mapsto \sum_{i=1}^{n} d\left(f\left(t, x_{i}\right), f\left(t, x_{i-1}\right)\right)
$$

is of the 1st class of Baire on $X^{n+1}$. Let $A_{n}$ be the set defined in Lemma 2.1 for fixed $r \geq 0$. By Lemma 2.2, $A_{n} \in \mathcal{T} \otimes \mathcal{B}\left(X^{n+1}\right)$. Moreover, for each $t \in T$ we have

$$
\left(A_{n}\right)_{t}=\left\{\left(x_{0}, \ldots, x_{n}\right) \in X^{n+1}: x_{0}<\cdots<x_{n}, \sum_{i=1}^{n} d\left(f\left(t, x_{i}\right), f\left(t, x_{i-1}\right)\right)>r\right\},
$$

and this set belongs to $F_{\sigma}\left(X^{n+1}\right)$. Since $X^{n+1}$ is $\sigma$-compact, $\left(A_{n}\right)_{t}$ is also $\sigma$ compact. By the Arsenin-Kunugui-Novikov Theorem, $\operatorname{Proj}_{T} A_{n} \in \mathcal{B}(T)$. Thus $v$ is Borel measurable.

Corollary 2.4. If $T$ is a Polish space, $f$ is Borel measurable, and for each $t \in T f(t, \cdot)$ is of bounded variation, then $v$ is Borel. 
Proof. Being of bounded variation, $f(t, \cdot)$ has at most countable set of discontinuity points (see e.g., [1],[5]). Consequently, $f(t, \cdot)$ is of the 1 st class of Baire (cf. [7, 34.VII]). Now we can apply Theorem 2.3.

Example 2.3 shows that the assumption of the bounded variation of $f(t, \cdot)$ is essential for the Borel measurability of $v$.

If we assume that $f$ is of the 1st class of Baire as a function of two variables, then we can strengthen the thesis of Theorem 2.3.

Theorem 2.4. If $T$ is metrizable and $\sigma$-compact, and $f$ is of the 1st class of Baire, then $v$ is of the 2nd class.

Proof. Fix $r \geq 0$, and let $A_{n}$ be such as in Lemma 2.1. Under our assumptions, the function $\mathrm{g}$ defined in the proof of Lemma 2.2 is of the 1st class of Baire on $T \times X^{n+1}$. Consequently, $A_{n} \in F_{\sigma}\left(T \times X^{n+1}\right)$. Since $T \times X^{n+1}$ is $\sigma$-compact, $A_{n}$ is $\sigma$-compact, and $\operatorname{Proj}_{T} A_{n}$ is also $\sigma$-compact. Hence, $\{t \in T: v(t)>r\} \in F_{\sigma}(T)$. Moreover, $\{t \in T: v(t)<r\}=T \backslash\{t \in$ $T: v(t) \geq r\}=T \backslash \bigcap_{k \in \mathbb{N}}\left\{t \in T: v(t)>r+\frac{1}{k}\right\} \in G_{\delta \sigma}(T)$. It completes the proof.

Remark 2.2. Example 2.3 shows that there is no analogous result for functions of the next classes of Baire. The function $f=\chi_{D}$ from this example is of the 2nd class of Baire, and $v$ is not Borel measurable. It follows from the fact that $D$ is a $G_{\delta}$-set in $[0,1]^{2}$, as a closed subset of $[0,1] \times P$.

\section{Continuity}

In this section we collect some simple observations concerning the continuity properties of the function $v$. We shall assume that $T$ is a topological space. First consider the following example:

Example 3.1. Continuous function $f$ such that $v$ is discontinuous.

Let $T=[0,1], X=(0,1], Y=\mathbb{R}$ and $f(t, x)=x^{t}$. Then $v(0)=0$ and $v(t)=1$ for $t>0$.

Note that the function $v$ in this example is lower semicontinuous.

Theorem 3.1. If $f$ is continuous in $t$, then $v$ is lower semicontinuous.

Proof. For each partition $\Pi=\left\{x_{0}, \ldots, x_{n}\right\}$ of $X$ the function

$$
t \mapsto \sum_{i=1}^{n} d\left(f\left(t, x_{i}\right), f\left(t, x_{i-1}\right)\right)
$$


is continuous. Hence, $v$ is lower semicontinuous, as the pointwise supremum of continuous functions.

As a consequence, we obtain the following well known property of the total variation:

Corollary 3.1. If $g_{k}, g: X \rightarrow Y$ and the sequence $\left(g_{k}\right)$ pointwise converges to $g$, then

$$
V(g, X) \leq \liminf _{k \rightarrow \infty} V\left(g_{k}, X\right)
$$

Proof. In order to apply Theorem 3.1, we put $T=\left\{\frac{1}{k}: k \in \mathbb{N}\right\} \cup\{0\}$, $f\left(\frac{1}{k}, x\right)=g_{k}(x)$ for $k \in \mathbb{N}$, and $f(0, x)=g(x)$. Then $v\left(\frac{1}{k}\right)=V\left(g_{k}, X\right)$ and $v(0)=V(g, X)$. By the lower semicontinuity of $v$ at $0, \liminf _{k \rightarrow \infty} v\left(\frac{1}{k}\right) \geq$ $v(0)$.

Now we give a condition equivalent to the continuity of the function $v$.

Theorem 3.2. Let $T$ be a compact metric space, $(Y, d)$ a metric space, and $f: T \times[a, b] \rightarrow Y$. Assume that $f(\cdot, x)$ is continuous for each $x \in[a, b]$, and $f(t, \cdot)$ is continuous of bounded variation for each $t \in T$. The function $v$ is continuous on $T$ if and only if the sequence $\left(\varphi_{n}\right)$ of functions $\varphi_{n}: T \rightarrow \mathbb{R}$ given by

$$
\varphi_{n}(t)=\sum_{k=1}^{2^{n}} d\left(f\left(t, a+(k-1) \frac{b-a}{2^{n}}\right), f\left(t, a+k \frac{b-a}{2^{n}}\right)\right), t \in T,
$$

is uniformly convergent on $T$.

Proof. Since for a fixed $t \in T$ the function $f(t, \cdot)$ is continuous on $[a, b]$, $v(t)=\lim _{n \rightarrow \infty} \varphi_{n}(t)$ (see [8]; the proof given there for the real-valued case also holds for functions with values in a metric space). ¿From the continuity of $f(\cdot, x)$ (for every $x$ ) it follows that the functions $\varphi_{n}$ are continuous on $T$.

By the above remarks, the proof of sufficiency is obvious. Let us show necessity. Observe that for $k \in\left\{1, \ldots, 2^{n}\right\}$ we have

$$
\begin{aligned}
& d\left(f\left(t, a+(k-1) \frac{b-a}{2^{n}}\right), f\left(t, a+k \frac{b-a}{2^{n}}\right)\right) \\
\leq & d\left(f\left(t, a+(2 k-2) \frac{b-a}{2^{n+1}}\right), f\left(t, a+(2 k-1) \frac{b-a}{2^{n+1}}\right)\right) \\
+ & d\left(f\left(t, a+(2 k-1) \frac{b-a}{2^{n+1}}\right), f\left(t, a+2 k \frac{b-a}{2^{n+1}}\right)\right) .
\end{aligned}
$$

Consequently, $0 \leq \varphi_{n} \nearrow v$, and $\varphi_{n}, v$ are continuous on $T$. Hence by the Dini theorem, $\left(\varphi_{n}\right)$ converges to $v$ uniformly on $T$. 
Let $(Y, d)$ be a metric space. Remind that a function $g:[a, b] \rightarrow Y$ is absolutely continuous if for any $\varepsilon>0$ there exists $\delta>0$ such that for any finite number of points $a \leq a_{1}<b_{1} \leq a_{2}<b_{2} \leq \cdots \leq a_{n}<b_{n} \leq b$ the condition $\sum_{i=1}^{n}\left(b_{i}-a_{i}\right)<\delta$ implies $\sum_{i=1}^{n} d\left(g\left(b_{i}\right), g\left(a_{i}\right)\right)<\varepsilon$. It appears that such functions have analogous properties as in the real-valued case. Recently V. V. Chistyakov and R. E. Svetic [3] obtained the following result.

Theorem 3.3. If $g:[a, b] \rightarrow Y$ is absolutely continuous, then for almost all $x \in(a, b)$ there exists the limit (called the metric derivative of $g$ at $x$ )

$$
\left|g^{\prime}\right|(x)=\lim _{h \rightarrow 0} \frac{d(g(x+h), g(x))}{h},
$$

and the following integral formula holds

$$
V(g,[a, b])=\int_{a}^{b}\left|g^{\prime}\right|(x) d x .
$$

This integral formula is an extension of the corresponding result for continuously differentiable functions with values in a normed space (cf. [1]).

For $f: T \times[a, b] \rightarrow Y$ absolutely continuous in the second variable we denote by $\left|f_{x}^{\prime}\right|(t, \cdot)$ the metric derivative of $f(t, \cdot)$. Hence,

$$
v(t)=\int_{a}^{b}\left|f_{x}^{\prime}\right|(t, x) d x .
$$

In order to apply this formula in the study of the continuity of $v$, we have to assume that the metric derivative $\left|f_{x}^{\prime}\right|$ depends continuously on $t$. But for fixed $x \in(a, b)$, the function $\left|f_{x}^{\prime}\right|(\cdot, x)$ may be defined not for all $t \in T$ (or even undefined). Therefore we shall assume that $\left|f_{x}^{\prime}\right|$ can be extended to a function $g$ defined on $T \times[a, b]$ which satisfies some regularity conditions. For example, for $f(t, x)=|t-x|$ the function $g \equiv 1$ is such an extension. More precisely, we impose the following assumption:

(A) The function $f(t, \cdot)$ is absolutely continuous for every $t \in T$, and there exists a function $g: T \times[a, b] \rightarrow \mathbb{R}$ continuous in $t$ and such that for each $t \in T, g(t, x)=\left|f_{x}^{\prime}\right|(t, x)$ for almost all $x \in[a, b]$.

In particular, the assumption (A) is satisfied if $Y$ is a normed space, $f$ is differentiable in the second variable and this derivative is continuous on $T \times[a, b]$.

Theorem 3.4. Let $T$ and $Y$ be metric spaces and $f: T \times[a, b] \rightarrow Y$. Assume (A). Moreover, assume that there exists an integrable function $h:[a, b] \rightarrow \mathbb{R}$ such that $g(t, x) \leq h(x)$ for all $(t, x) \in T \times[a, b]$. Then $v$ is continuous. 
Proof. Let $t_{0} \in T$ and a sequence $\left(t_{n}\right) \subset T$ converging to $t_{0}$ be arbitrary but fixed. By the Lebesgue Dominated Convergence Theorem we obtain

$$
\begin{aligned}
\lim _{k \rightarrow \infty} v\left(t_{k}\right) & =\lim _{k \rightarrow \infty} \int_{a}^{b}\left|f_{x}^{\prime}\right|\left(t_{k}, x\right) \mid d x=\lim _{k \rightarrow \infty} \int_{a}^{b} g\left(t_{k}, x\right) d x \\
& =\int_{a}^{b} g\left(t_{0}, x\right) d x=v\left(t_{0}\right) .
\end{aligned}
$$

It shows the continuity of $v$.

In the next theorem we prove the Lipschitz continuity of the function $v$.

Theorem 3.5. Let $(T, \varrho)$ and $(Y, d)$ be metric spaces, and $f: T \times[a, b] \rightarrow Y$. Assume (A). Moreover, suppose that $g(\cdot, x)$ satisfies the Lipschitz condition; i.e., $|g(s, x)-g(t, x)| \leq h(x) \varrho(s, t)$ for all $s, t \in T, x \in[a, b]$, where $h:[a, b] \rightarrow$ $\mathbb{R}$ is an integrable function. Then the function $v$ is Lipschitzian.

Proof. Fix $s, t \in T$. Then

$$
\begin{aligned}
|v(s)-v(t)| & =\left|\int_{a}^{b}\right| f_{x}^{\prime}\left|(s, x) d x-\int_{a}^{b}\right| f_{x}^{\prime}|(t, x) d x| \\
& =\left|\int_{a}^{b} g(s, x) d x-\int_{a}^{b} g(t, x) d x\right| \leq \int_{a}^{b}|g(s, x)-g(t, x)| d x \\
& \leq\left(\int_{a}^{b} h(x) d x\right) \varrho(s, t) .
\end{aligned}
$$

Remark 3.1. Since we use the Lebesgue integral, it suffices if the estimations in the assumptions of Theorems 3.4 and 3.5 hold for almost all $x \in[a, b]$.

Acknowledgement. We are grateful to V. V. Chistyakov (Nizhny Novgorod, Russia) for letting us know Theorem 3.3 from his joint paper with R. E. Svetic [3] and for helpful comments. The research of the second and third author was supported by the Department of Mathematics of the Silesian University in Katowice.

\section{References}

[1] V. V. Chistyakov, On mappings of bounded variation, J. Dynam. and Control Syst., 3(2) (1997), 261-289. 
[2] V. V. Chistyakov and A. Nowak, Regular Carathéodory-type selectors under no convexity assumptions, submitted.

[3] V. V. Chistyakov and R. E. Svetic, On the differentiation of multifunctions of bounded variation, (in preparation).

[4] J. P. R. Christensen, Topology and Borel Structures, North-Holland, Amsterdam, 1974.

[5] H. Federer, Geometric Measure Theory, Springer-Verlag, 1969.

[6] A. S. Kechris, Classical Descriptive Set Theory, Springer-Verlag, 1994.

[7] K. Kuratowski, Topology, vol. I, Academic Press and PWN, 1966.

[8] I. P. Natanson, Theory of Functions of a Real Variable, Frederic Ungar Publishing Co, New York, 1964 (Russian edition: Moskva 1950).

[9] T. Neubrunn, Quasi-continuity, Real Anal. Exchange, 14 (1988-1989), 259-306. 